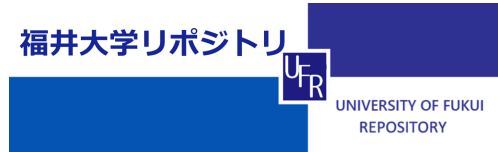

St abi I i zat i on and modul at i on of the out put power of submilli meter wave gyrotron

\begin{tabular}{|c|c|}
\hline 著者 & OGAWA I, I DEHARA T, UI M M TSUDO S, FORSTER W \\
\hline $\begin{array}{l}\text { j our nal or } \\
\text { publ i cat i on } \mathrm{titl} \text { e }\end{array}$ & Fusi on Engi neeri ng and Desi gn \\
\hline vol une & 53 \\
\hline nunber & 1- 4 \\
\hline page $r$ ange & $571-576$ \\
\hline year & $2001-01$ \\
\hline URL & ht t p: //hdl . handl e. net /10098/1553 \\
\hline
\end{tabular}




\title{
Stabilization and modulation of the output power of submillimeter wave gyrotron
}

\author{
I. Ogawa ${ }^{a}$, T. Idehara ${ }^{b}, \mathbb{M}$. Ui ${ }^{a}$, S. Mitsudo ${ }^{b}, W$. Förster ${ }^{c}$ \\ ${ }^{a}$ Faculty of Engineering, Fukui University, 3-9-1 Bunkyo, Fukui 910-8507, Japan \\ ${ }^{b}$ Research Center for Development of Far-Infrared Region, Fukui University, Fukui 910-8507, Japan \\ - Institut Für Plasmaforschung, Universität Stuttgart, Pfaffenwaldring 31, D-70569 Stuttgart, Germany
}

\begin{abstract}
High frequency, medium power gyrotrons (Gyrotron FU series) have been developed at the Fukui University as radiation sources convering a broad frequency band from millimeter to submillimeter wave region. We have performed cw operation of the Gyrotron FU IV. However, the output power was not stable (approximately several tens $\%$ ) due to the fluctuation of the cathode potential $(\sim 40 \mathrm{~V})$. The output power was stabillzed within several percent by controlling the anode potential. Such a technique was also used for amplitude modulation of the gyrotron output. Improved stabilization of output power and effective modulation will be achieved by stabilizing the cathode potential.
\end{abstract}

Keywords: Gyrotron; Stabilization; Modulation

\section{Introduction}

Up to now, molecular vapour lasers $[1,2]$ and backward-wave oscillators [3] have been the principal power sources in the submillimeter wavelength range. However, their output powers are restricted to $0.5 \mathrm{~W}$ or $10 \mathrm{~mW}$.

On the other hand, gyrotrons are suitable not only as high power sources $[4,5]$ but also as high frequency sources. High frequency gyrotrons have already been used as millimeter to submillimeter wave sources for plasma scattering measurements [6], electron spin resonance (ESR) experiments, etc. Such gyrotrons operate on fundamental, sec- ond, and even third harmonics of the electron cyclotron resonance under high magnetic field exceeding $10 \mathrm{~T}$. In addition, $\mathrm{cw}$ operation with output powers of several tens of watts has been achieved by several high frequency gyrotrons at the University of Sydney and by one of the submillimeter wave gyrotrons (Gyrotron FU IV) at Fukui University.

High frequency gyrotrons have many advantages (such as frequency tunability) compared to conventional sources and are also the most promising candidates as high power sources. For many applications of such sources, the stability of the output power is very important. It can be achieved by stabilizing the cathode potential and by a proper control of the anode voltage. We 
investigated both approaches and the results from our experiments are presented in this paper.

\section{Development of high fingegrency gyrotrons}

We have developed high frequency, medium power gyrotrons covering a broad frequency

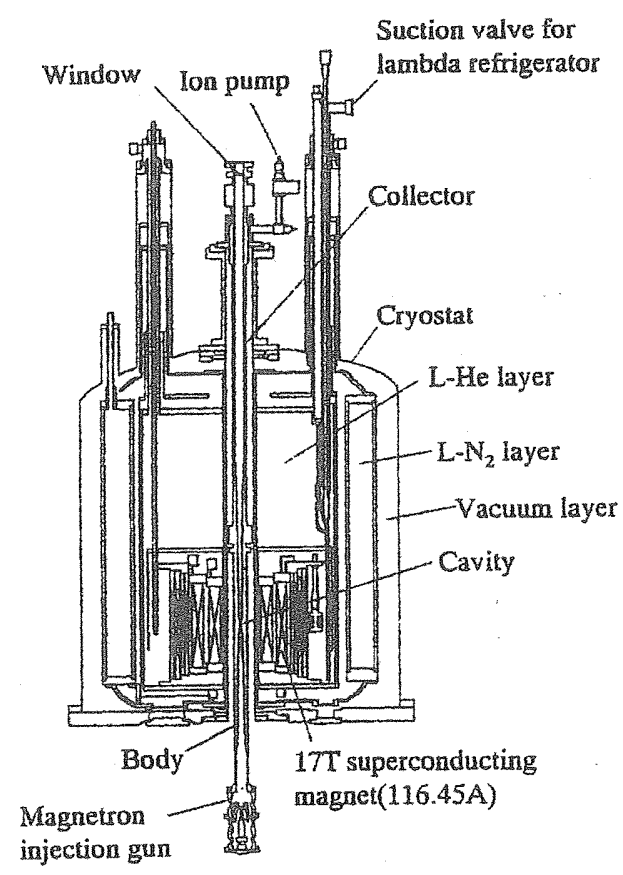

Fig. 1. A schematic drawing of Gyrotron FU IV A.

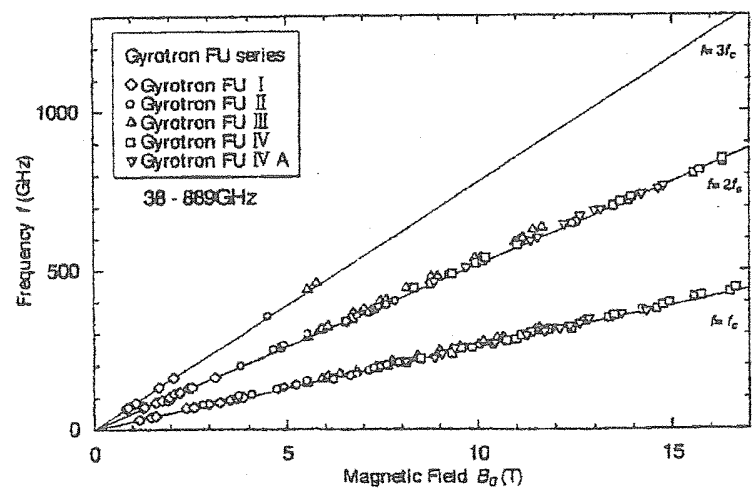

Fig. 2. Frequencies achieved up to now by Gyrotron FU series as a function of field intensity $B_{0}$. Solid lines show the fundamental, the second harmonic, and the third harmonic resonances.

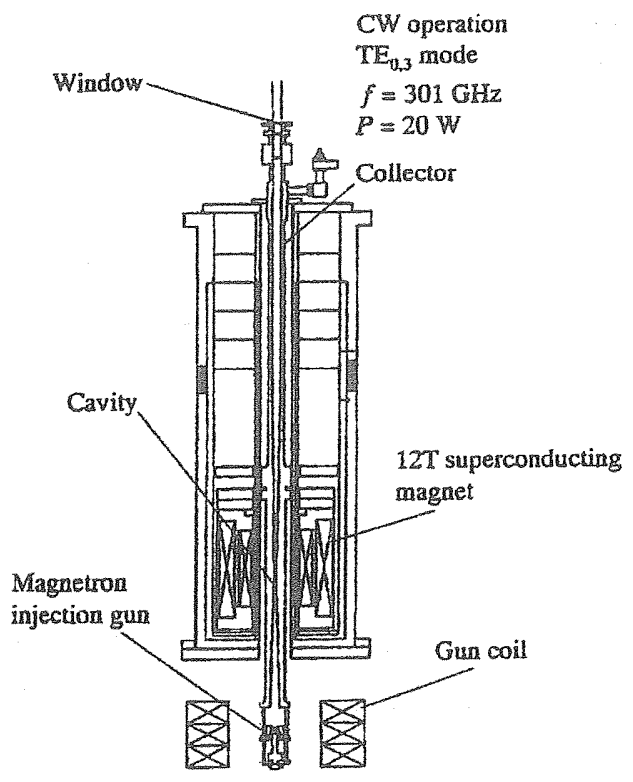

Fig. 3. A schematic drawing of Gyrotron FU IV.

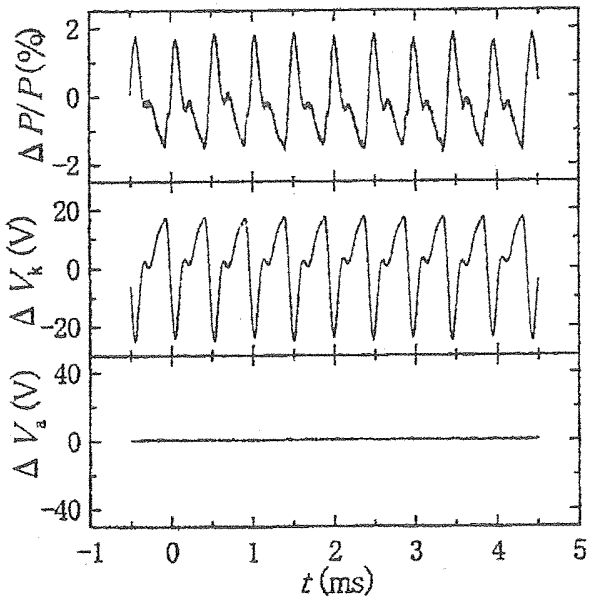

Fig. 4. Time evolution of operation parameters: $\triangle P$ fluctuation of gyrotron output power, $\Delta V_{\mathrm{k}}$ that of cathode potential and $\Delta V_{\text {a }}$ that of anode potential.

range from millimeter to submillimeter wavelength region. Our 'Gyrotron FU series' consists of eight gyrotrons. A schematic drawing of the latest gyrotron, Gyrotron FU IV A [7], is shown in Fig. 1. The gyrotron consists of a $17 \mathrm{~T}$ superconducting magnet, magnetron injection gun (MIG), resonant cavity, collector and a sealed-off tube. In order to improve the mode separation, 
we use a narrow cavity whose diameter is 3.23 min.

All frequencies achieved up to now by Gyrom tron FU series are summarized as a function of magnetic field intensity $B_{0}$ in Fig. 2. Solid lines

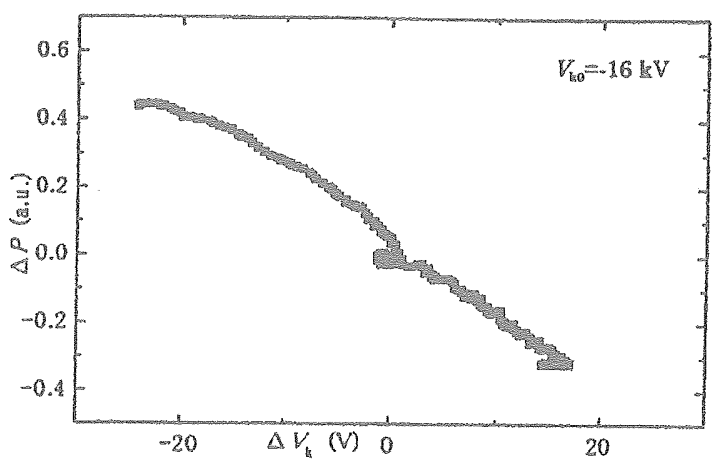

Fig. 5. Correlation between cathode potential fuctuations and output power Iluctuations.

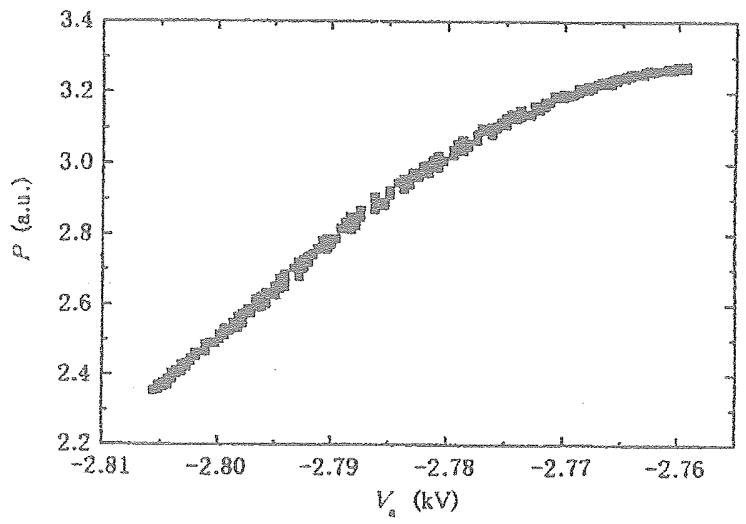

Fig. 6. Correlation between anode potential and output power (after the implementation of a low-pass fiter).

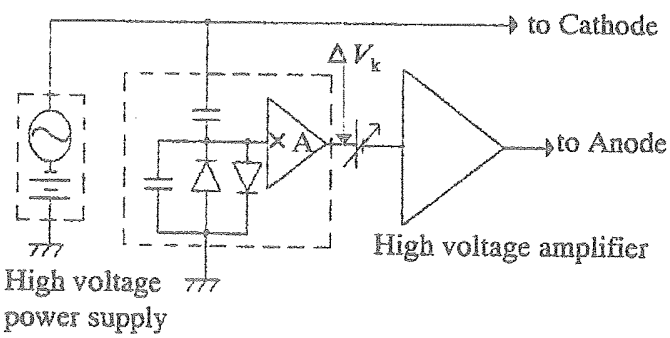

Fig. 7. A circuit for producing anode voltage to stabilize the output power.

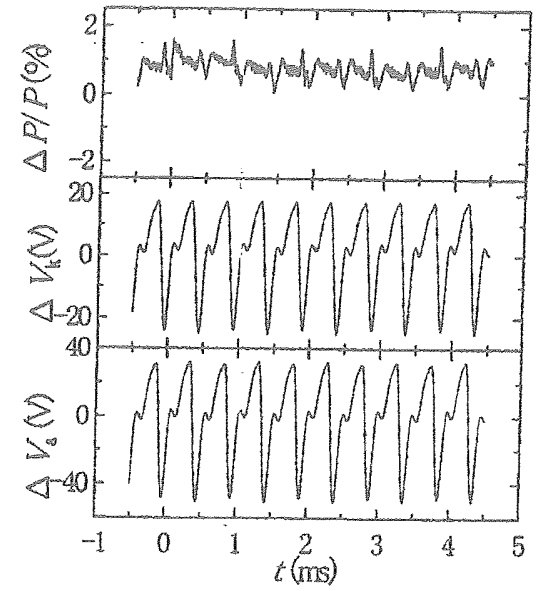

Fig. 8. Time evolution of operation parameters: $\Delta P$ fluctuation of gyrotron output power, $\Delta V_{k}$ that of cathode potential and $\Delta V_{\text {a }}$ that of anode potential.

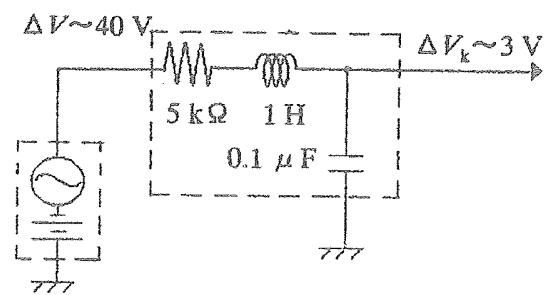

Fig. 9. A smoothing circuit to suppress the cathode potential fuctuation.

represent the fundamental $(N=1)$, the second $(N=2)$, and the third harmonic $(N=3)$ resonances. Frequency step twnability from 38 to 889 $\mathrm{GHz}$ is achieved for the fundamental, second, and third harmonic operation.

Gyrotron FU IV A achieved the highest frequency of $889 \mathrm{GHz}$ for single mode operation on the $\mathrm{TE}_{8,6,1}$ cavity mode at the second harmonic $(N=2)$. In the case of Gyrotron FU IV A, the output power ranges from several hundred watts at the fundamentals to several ten watts at second harmonics.

\section{Stabilizgation of the gyrotron ourpune by controlling the amode potentid}

We have successfully performed cw operation of the submillimeter wave gyrotron, Gyrotron FU 
IV [8]. This gyrotron consists of sealed-off tube and magnet system shown in Fig. 3. The superconducting magnet produces the main field in the cavity region whose intensity can be raised up to $12 \mathrm{~T}$. Three subsidiary copper field coils are used in the electron gun region. Both fields can be adjusted independently to control the formation of the electron beam in the MIG.

The cw operation (TE 03 mode, $f=301 \mathrm{GHz}$, $P=20 \mathrm{~W}$ ) is obtained under the following conditions: magnetic field intensity in the cavity region $B_{0}=10.8 \mathrm{~T}$, cathode potential $V_{\mathrm{k}}=-16 \mathrm{kV}$, anode potential $V_{\mathrm{a}}=-2.9 \mathrm{kV}$. The evolution of operation parameters with time is shown in Fig. 4. The fluctuations of the output power correlate with that of the cathode potential. When the cathode potential decreases to the minimum value, the output power reaches its maximum. The change of the output power as a function of the variation of the cathode potential is shown in
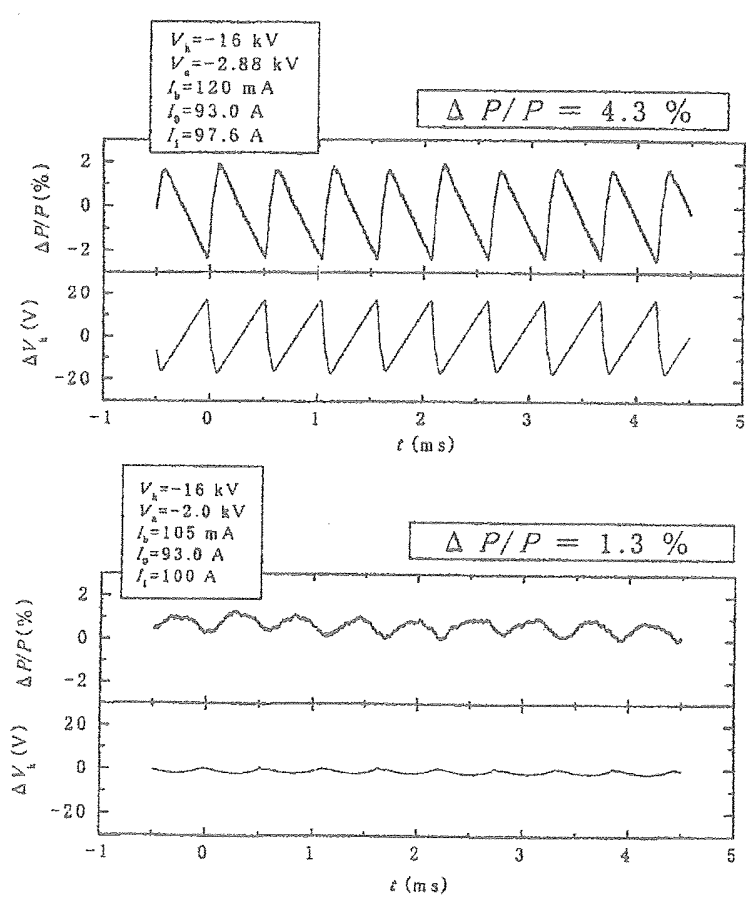

Fig. 10. Time evolution of operational parameters: $\Delta P$ fluctua. tion of gyrotron output power, $\Delta V_{\mathrm{k}}$ that of cathode potential: (a) without a smoothing circut; and (b) with a smoothing circuit.
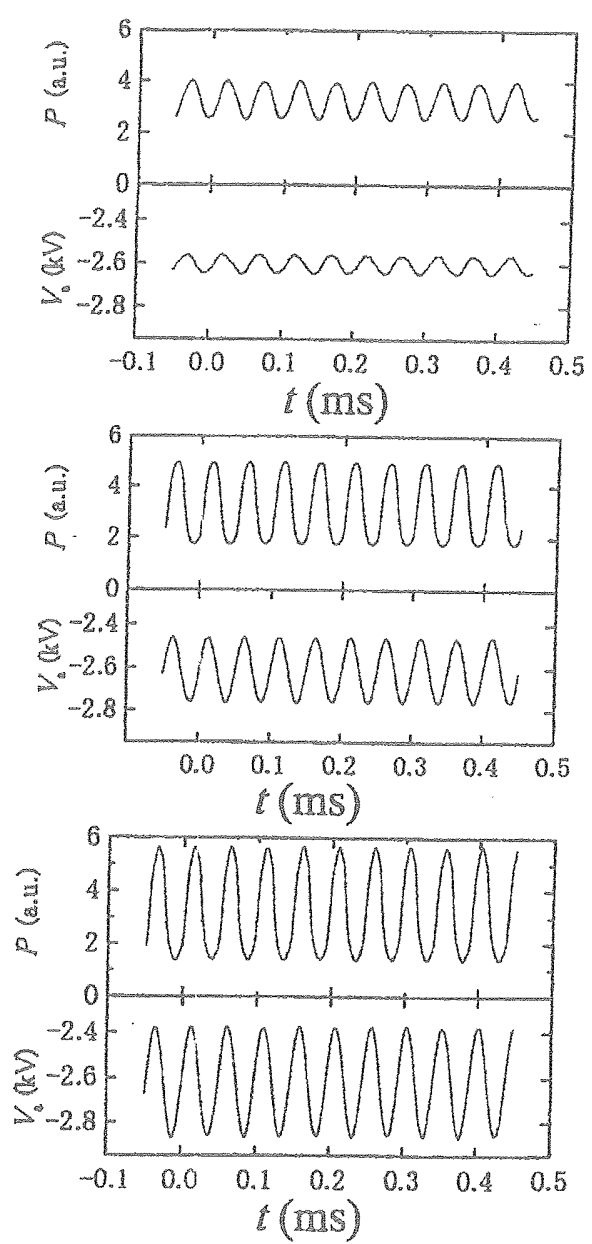

Fig. 11. The evolution of operational parameters: $P$ gyrotron output power, $V_{\mathrm{a}}$ anode potential: (a) $\Delta V_{\mathrm{a}} / Y_{\mathrm{a}}=1.8 \times 10^{-2}$; (b) $\Delta V_{\mathrm{a}} / V_{\mathrm{a}}=5.8 \times 10^{-2}$; (c) $\Delta V_{\mathrm{a}} / V_{\mathrm{a}}=9.0 \times 10^{-2}$.

Fig. 5. It can be seen that at the specific operational conditions the output power decreases monotonically with the increase of the cathode potential. On the other hand, the output power increases monotonically with the increase of the anode potential (Fig. 6). Therefore, the output power may be stabilized by controlling the anode potential using the fluctuations of the cathode potential as a feedback signal. The characteristic shown in Fig. 6 was obtained after the implementation of a low-pass filter which significantly reduces the output power fluctuations. The circuit, used for control of the anode potential is shown 
in Fig. 7. The fiuctuations of the cathode potential were measured using a divider made from capacitors. Our experience shows that it is possible to arrive at the optimal condition by just changing the amplifier's gain. As a result of the implementation of this approach we succeeded to reduce the fluctuation level from 3.7 to $1.5 \%$ (Fig. 8). By further optimization of the stabilization we hope to decrease the fluctuations down to $0.1 \%$.

\section{Stabilization of the gyrotron output by stabilinzing the crathode potentidal}

In order to suppress the fluctuation level of the cathode potential, the high voltage power supply is equipped with a smoothing circuit consisting of a resistor, an induction coil and a capacitor (Fig. 9). The time evolution of output power and cathode potential is shown in Fig. 10. The fluctuation level was decreased from 40 to $3 \mathrm{~V}$ by introducing the smoothing circuit. Accordingly, the fluctuations of the output power were decreased from 4.3 to $1.3 \%$, respectively.

\section{Amplitude moduldation}

We have already demonstrated amplitude modulation of gyrotron output in pulse operation [9]. The modulation is based on the relation between the output power and the anode potential as shown in Fig. 6. The results for the experimental conditions are illustrated in Fig. 11. As the modulation level of anode potential increases, that of the output power also increases. The depth of amplitude modulation is correlated with the modulation level of anode potential (Fig. 12). Such a relation doesn't depend on modulation frequency and suggests the possibility to attain higher modulation frequencies.

\section{Conclusionns}

High frequency, medium power gyrotrons (Gy. rotron FU series) achieved the highest frequency of $889 \mathrm{GHz}$ for single mode operation at the second harmonic $(N=2)$ and the frequency steptunability from 38 to $889 \mathrm{GHz}$ using the fundar mental, second, and third harmonic operation. Output powers of several hundred watts at the fundamentals and several ten watts at second harmonics have been achieved.

As a result of the feedback control of the anode potential we succeeded to reduce the fluctuation level of the cw output power $(f=301 \mathrm{GHz}, P=$ $20 \mathrm{~W})$ from 3.7 to $1.5 \%$. The fluctuation level could be decreased down to $0.6 \%$ by reducing Ifuctuation level of the cathode potential $<3 \mathrm{~V}$.

As a result of the control of the anode potential we succeded to modulate the gyrotron output with a modulation frequency up to $20 \mathrm{kHz}$.

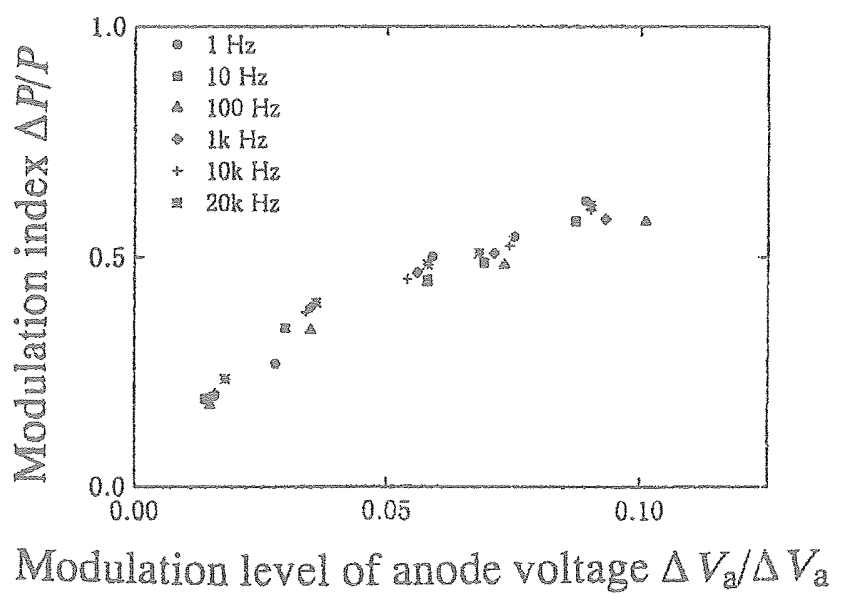

Fig. 12. Depth of the amplitude modulation as a function of modulation level of the anode potential. 


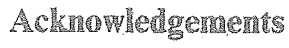

The authors wish to thank Dr $\mathbf{S}$. Sabchevsky from the Research Center for Development of Far-Infrared Region, Fukui University for his invaluable discussions. This work was carried out as a collaboration between Fukui University, the National Institute for Fusion Science and Universitat Stutegrart, Germany and was partially supported by a Grant-in-Aid from the Ministry of Education, Science and Culture of Japan and the Murata Science Foundation.

\section{References}

[1] A. Semet; A. Mase, W.A. Peebles, N.C. Luhmann, Ir, S. Zweben, Phys. Rev. Lett. 45 (1980) 445 .
[2] K. Kawahata, T. Tetsuka, 3. Fujita, M. Nagatsu, H. Ohnish, S. Okajina, T. Wasaki, Int. J. Infrared Millmeter Waves 9 (1988) 655 .

[3] I. Morino, M. Fabian, H. Takeo, K. Yamada, I. Mol Spectrosc. 185 (1997) 142.

[4] T. Idehara, T. Tatsukawa, I. Ogawa, Y. Shimizu, N. Nishida, K. Yoshida, Procedings of the Third International Conference on Strong Microwave in Plasmas, vol. 2 Moscow, Russia, 1997, p. 634.

[5] K.D. Hong, G.F. Brand, T. Idehara, A. Appl. Phys. 74 (1993) 5250

[6] I. Ogawa, M. Twata, T. Idebara, K. Kawahata, M. Iguchi, A. Ejiri, Fus. Eng, Des. $34-35$ (1997) 435.

[7] T. Luehara, N. Nishida, K. Yoshida, I. Ogawa, T. Tatsukawa, D. Wagner, G. Gantenbein, W. Kasparek, M. Thumm, Int. J. Infrared Millimeter Waves 19 (1998) 919.

[8] T. Idehara, $\mathbb{K}$. Yoshida, N. Nishida, I. Ogawa, M. Pereyaslavets, T. Tatsukawa, Int. I. Infrared Millimeter Waves 19 (1998) 793.

[9] T. Idehara, $Y$. Shimizu, S. Makino, K. Tchkawa, T. Tatsukawa, 1. Ogawa, G.F. Brand, Int. J. Infrared Millimeter Waves 18 (1997) 391. 